\title{
First Candle Receives Grant from W.K. Kellogg Foundation to Address Racial Disparity in Infant Mortality Rates
}

\section{Barb Himes, IBCLC}

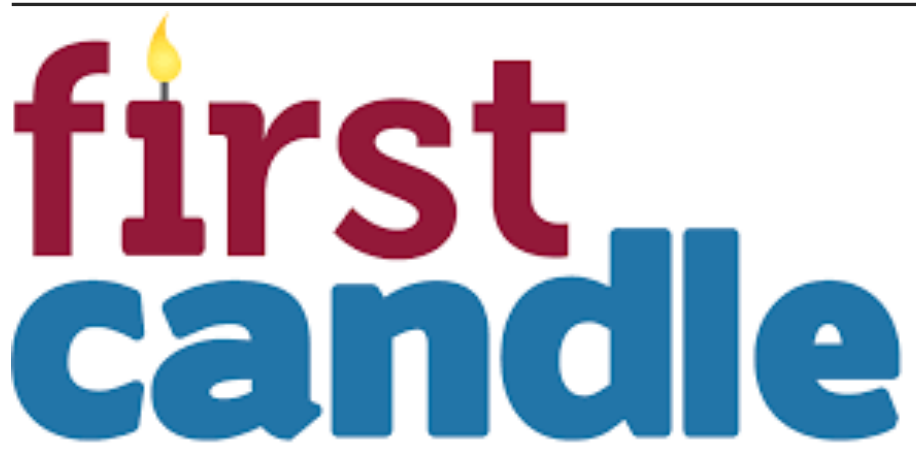

Saving babies. Supporting families.

First Candle's efforts to support families during their most difficult times and provide new answers to help other families avoid the tragedy of the loss of their baby are without parallel.

Program Will Address Role of Implicit Bias in Safe Sleep Messaging

"This work is part of a larger set of

First Candle goals aimed at addressing the impact of implicit bias, systemic racism and the social determinants of health on high rates of infant deaths among families of color."

First Candle, the national non-profit committed to ending Sudden Unexpected Infant Deaths (SUID), has announced it is undertaking an initiative to collect input from communities of color to and share with the American Academy of Pediatrics (AAP) as it updates the Safe Sleep Guidelines, for release in 2021. This work will be supported by a $\$ 100,000$ grant from the W.K. Kellogg Foundation, which will be directed to communities in Michigan.
This work is part of a larger set of First Candle goals aimed at addressing the impact of implicit bias, systemic racism and the social determinants of health on high rates of infant deaths among families of color.

Sudden Infant Death Syndrome (SIDS) and accidental suffocation and strangulation in bed (ASSB) are classified under SUID and account for 3,600 infant deaths in the U.S. every year. The rate of SUID is more than twice as high for Black babies as white babies. ASSB has increased by $115 \%$ over the past decade and can be prevented by adopting the AAP Safe Sleep Guidelines. The guidelines include recommendations that families always place a baby alone in a crib or another surface designed for infants, on a firm, flat mattress in a supine position with nothing in the area including pillows, blankets, stuffed animals, fluffy bumpers or loose clothing.

According to First Candle, the risk of SIDS can be dramatically reduced by following these guidelines as well as breastfeeding for the first six months to a year of life, but these guidelines are not widely followed across all communities.

To better understand the issues surrounding adoption of the Safe Sleep Guidelines and to facilitate community-driven campaigns that eliminate the racial bias in messaging, First Candle will convene Community Task Forces in three states, including Michigan. The Task Forces will be comprised of parents, grandparents, inhome health care providers, social service agencies and doulas who will share their thoughts on the Safe Sleep Guidelines, the challenges and objections that exist in adopting them and the role systemic racism has played in accessing this life-saving information.

This information will be shared with the AAP as it develops the updated 2021 Safe Sleep Guidelines. Upon release of the new Guidelines, the Community Task Forces will create solutions-oriented education and outreach campaigns consistent with the lived experiences of the community to facilitate adoption of the revised Guidelines. Simultaneously, First Candle will continue the implicit bias training program, Straight Talk for Infant Safe Sleep, that it delivers to hospitals, health care providers and social service agencies to help foster dialogue between families and providers on safe sleep and breastfeeding.

"At First Candle we are committed to addressing the alarming rates of Black infant deaths from SUID, said First Candle CEO Alison Jacobson. "We want every baby to reach his or her first birth-

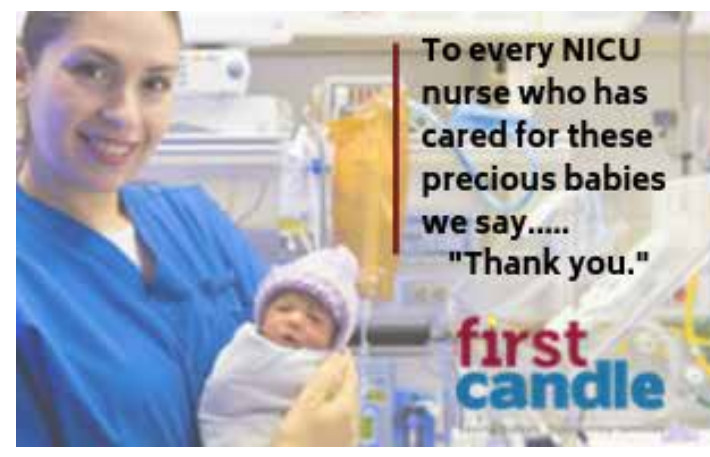

\section{Did you know that premature and low birth weight babies have a $4 \times$ greater risk for SIDS?}

At First Candle we're educating parents, grandparents and caregivers about safer sleep to make sure all babies reach their first birthday. Learn more at firstcandle.org 
day and the first step is eliminating racial disparity. We know the answers to the challenges of adopting the Safe Sleep Guidelines lie in the community and it's our job to listen, learn and support.

"We are proud to align with organizations such as Black Mothers' Breastfeeding Association, Black Mamas Matter and Moms Rising to end bias that exists within the healthcare system and save babies' lives. With the support of the W.K. Kellogg Foundation, we know we can finally drive down the number of infant deaths. We are incredibly grateful for this grant."

In addition to Michigan, Community Task Forces are being convened in Connecticut and Georgia.

\section{References:}

1. http://www.aap.org/

2. https://firstcandle.org/aaps-infant-safe-sleep-recommendations/

3. https://firstcandle.org/straight-talk-for-infant-safe-sleep/

Disclosure: The author is the Director of Education and Bereavement Services of First Candle, Inc., a Connecticut not for profit 501c3 corporation.

NT

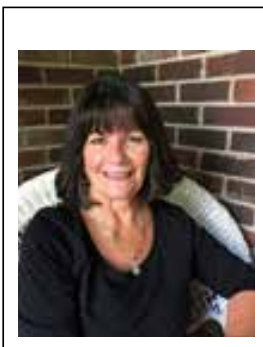

Corresponding Author

Barb Himes, IBCLC

Director of Education and Bereavement Services

First Candle

49 Locust Avenue, Suite 104

New Canaan CT 06840

Telephone: 1-203-966-1300

For Grief Support: 1-800-221-7437

barb@firstcandle.org

www.firstcandle.org

\section{Readers can also follow NEONATOLOGY TODAY via our Twitter Feed @NEOTODAY}
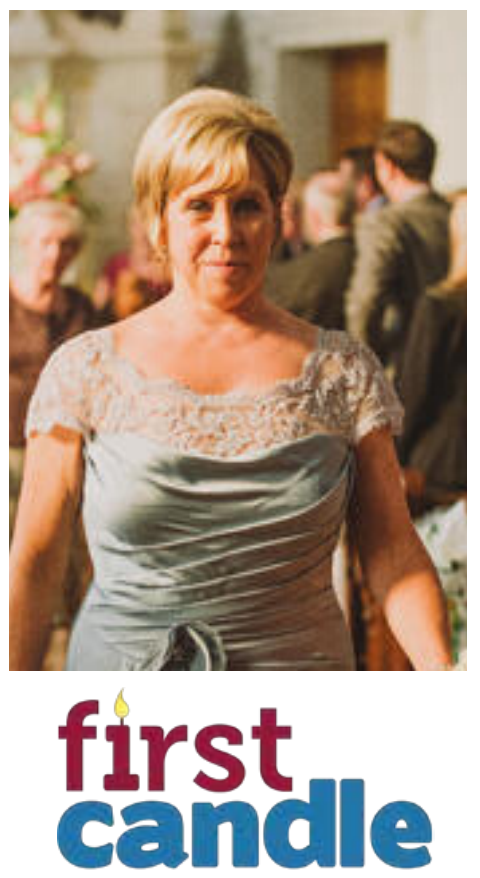

Saving babies. Supporting families
In January, heaven

gained a new angel -

Laura Reno.

Laura was a SIDS mom and a guiding force at First Candle.

She worked tirelessly to end SIDS and was a source of comfort for many of our berieved families.

Laura will be greatly missed.

\section{Oo National Perinatal Association PERINATAL SUBSTANCE USE}

nationalperinatal.org/position

www.nationalperinatal.org/Substance_Use

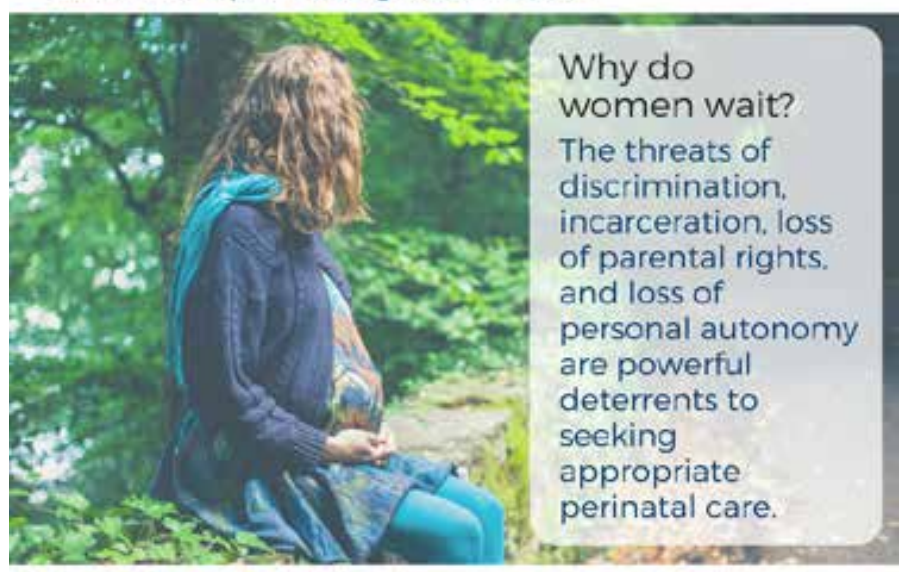

Educate. Advocate. Integrate.
Why do women wait?

The threats of discrimination. incarceration. loss of parental rights and loss of personal autonomy are powerful deterrents to seeking apropriate

\section{Time is precious, just like your patients.}

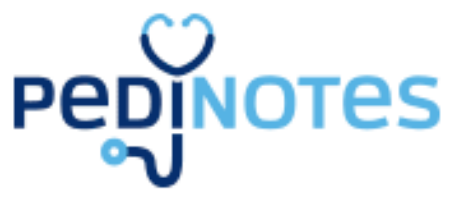

